\title{
Nut consumption and the impact on gut microbiome and gut function in healthy people: a systematic review of randomised controlled trials
}

\author{
A. Creedon, E. Hung, S. Berry and K. Whelan \\ Department of nutritional sciences, Kings College London, London SE1 9NH
}

The benefits of nut consumption on cardiometabolic health have been demonstrated in numerous systematic reviews. Nuts contain fibre, unsaturated fatty acids and polyphenols that may impact gut microbiome composition ${ }^{(1,2)}$. In vitro studies suggest a prebiotic effect of nuts on the gut microbiome ${ }^{(3)}$. Despite containing compounds that impact gut microbiome, gut function (e.g. stool output, transit time) and gut symptoms, there has been no systematic review investigating the effect of nuts on these aspects. The aim of this study is to perform a systematic review and meta-analysis of randomised controlled trials (RCTs) investigating the impact of nut consumption on gut microbiome, function and symptoms.

The systematic review was performed according to the guidelines of PRISMA and the Cochrane collaboration and was registered on PROSPERO (CRD4201913816). Five electronic databases were searched using predefined keywords, together with handsearching conference abstracts from the previous five years, clinical trials databases, and back-searching reference lists of eligible studies. Key stakeholders were contacted to identify potentially eligible unpublished studies. Eligible studies were RCTs in which participants were administered tree nuts or peanuts at a minimum dose of $7 \mathrm{~g} / \mathrm{d}$ for a minimum duration of 1 week in comparison to control, and where any outcome related to gut microbiome, gut function or gut symptoms was assessed. Two reviewers independently screened papers identified in the search, performed data extraction and risk of bias assessment.

The searches identified 2958 articles, of which 17 papers were reviewed in full. In total, seven RCTs were eligible, including 366 participants, investigating almonds $(n=5)$, walnuts $(n=2)$ and pistachios $(n=1)$. Doses ranged from $42-84 \mathrm{~g} / \mathrm{d}$ for $18-56 \mathrm{~d}$. The outcomes measured included gut microbiome composition $(n=6)$, stool output $(n=2)$ and gut symptoms $(n=2)$. No studies evaluated gut transit time. Of the RCTs reporting gut microbiome, one reported an increase in Bifidobacteria following walnut consumption, two reported a decrease in the genus following walnut or almond consumption and three reported no difference or did not report Bifidobacteria. One RCT reported an increase in $\alpha$-diversity following almond consumption, while 5 RCTs did not. Two RCTs reported significant alterations in $\beta$-diversity and three reported no change. Almond consumption was reported to decrease diarrhoea syndrome in one RCT, however a second RCT reported an increase in stool frequency in patients with diarrhoea-predominant irritable bowel syndrome. The majority of studies $(n=5)$ measured gut health as secondary outcomes. Risk of bias was unclear for the majority of studies due to inadequate reporting. Outcome data will undergo meta-analysis.

Nuts may impact the gut microbiome and stool frequency; however the consistency, direction and size of these effects is unclear. This review highlights the need for appropriately designed RCTs assessing the impact of nut consumption on gut microbiome and markers of gut health as the primary outcome.

1. Wolters M, Ahrens J, Romaní-Pérez M et al. (2018) Clin Nutr (In press).

2. Marin L, Miguélez EM, Villar CJ et al. (2015) Biomed Res Int 2015, 902-15.

3. Lamuel-Raventos RM, St Onge MP (2017) Crit Rev Food Sci Nutr 57, 3154-63. 